\title{
Pancreatitis aguda en pediatría como complicación de síndrome inflamatorio multisistémico asociado a COVID-19: un caso clínico
}

\author{
Acute pancreatitis in pediatrics as a complication of multi-systemic \\ inflammatory syndrome associated with COVID-19: A case report
}

\author{
Dr. Diego Carletti ${ }^{a}$, Dra. Daniela Porte ${ }^{a}$ Dr. Sebastián Masnicoffb, Dra. Flavia Colombo ${ }^{b}$ y \\ Dra. Mariana Romano
}

\begin{abstract}
RESUMEN
En abril de 2020, en informes provenientes del Reino Unido se notificó una presentación en niños similar a la enfermedad de Kawasaki incompleta o al síndrome de choque tóxico asociados con la enfermedad por el nuevo coronavirus (COVID-19). Desde entonces, ha habido informes de niños afectados de manera similaren otras partes del mundo. En la actualidad, la afección se ha denominado síndrome inflamatorio multisistémico asociado a COVID-19 en niños (SIM-C). Por otra parte, se notificaron casos de pancreatitis aguda, en su mayoría en pacientes adultos, en el contexto de la infección aguda por el coronavirus 2 causante del síndrome respiratorio agudograve(SARS-COV-2), causada por efectos citopáticos directos o respuestas celulares indirectas sistémicas inflamatorias e inmunomediadas. Sin embargo, son escasas las notificaciones en las que se describe el cuadro de pancreatitis aguda durante el SIM-C. Se describe aquí la presentación clínica, el tratamiento y la evolución de una paciente de 9 años que presentó un cuadro de pancreatitis aguda en el contexto del SIM-C.

Palabras clave: pancreatitis, infecciones por coronavirus, pediatría.
\end{abstract}

\begin{abstract}
In April 2020, UK studies informed a condition in children similar to incompleteKawasaki disease or toxic shock syndrome. From that time onwards, papers on children suffering from similar conditions have been published in different parts of the world. Today the disease is named multisystem inflammatory syndrome in children (MIS-C) associated with Covid-19.

Acute pancreatitis was reported mainly in adult patients with acuteSARS-COV-2 infection developing direct cytopathiceffect or immune-mediated and systemic inflammatory indirect cellular responses. However, there are only a few studies, which describe the acute pancreatitis case during MIS-C period. The present article describes the clinical presentation, therapy and evolution of a 9 years-old female patient developing an acute pancreatitis case suffering from MIS-C.

Key words: pancreatitis, coronavirus infections, pediatrics.
\end{abstract}

a. Servicio de Clínica Pediátrica.

b. Servicio de Clínica Pediátrica.

Hospital Municipal de Agudos Dr. Leónidas Lucero,

Bahía Blanca, Argentina.

Correspondencia:

Dr. Diego Carletti: diego-carletti@hotmail.com

Financiamiento: Ninguno.

Conflicto de intereses: Ninguno que declarar.

Recibido: 19-1-2021

Aceptado: 15-4-2021 http: / / dx.doi.org/10.5546/ aap.2021.e554

Cómo citar: Carletti D, Porte D, Masnicoff S, Colombo F, Romano M. Pancreatitis aguda en pediatría como complicación de síndrome inflamatorio multisistémico asociado a COVID-19: un caso clínico. Arch Argent Pediatr 2021;119(5):e554-e558.

\section{INTRODUCCIÓN}

En abril de 2020, en informes provenientes del Reino Unido, se notificó una presentación en niños similar a la enfermedad de Kawasaki incompleta (EK) o al síndrome de choque tóxico, con comienzo de 4 a 6 semanas posteriores a la infección por SARS-COV-2. ${ }^{1}$ Los informes de niños afectados de manera similar en otras partes del mundo fueron en aumento, hasta que se decidió denominar al cuadro como síndrome inflamatorio multisistémico post-COVID en niños (SIM-C).

La mayoría de los pacientes se presentan con fiebre de tres a cinco días, y los síntomas gastrointestinales (dolor abdominal, vómitos, diarrea) son frecuentes. ${ }^{2}$ Puede haber afectación multiorgánica, con alteración de las enzimas hepáticas y cardíacas, alteración del coagulograma y aumento de los reactantes de fase aguda como la proteína $C$ reactiva (PCR), eritrosedimentación (ERS), dímero $\mathrm{D}$ y ferritina, pero no se describió compromiso pancreático en un comienzo. ${ }^{3}$ Se describe una paciente que presentó un cuadro de pancreatitis aguda en el contexto del síndrome inflamatorio multisistémico post-COVID-19.

\section{CASO CLÍNICO}

Paciente de sexo femenino de 9 años y 11 meses de edad, con antecedentes de obesidad, que es llevada a la consulta por presentar 5 días de fiebre (con un máximo de $38,5^{\circ} \mathrm{C}$ ) con escasa respuesta a antitérmicos, astenia, tos y dolor abdominal. A las 48 horas, aparece un exantema 
maculopapular en miembros inferiores y tronco, diarrea e inyección conjuntival. Como antecedente epidemiológico, presentaba familiares con infección por el coronavirus 2 causante del síndrome respiratorio agudo grave (SARS-CoV-2) un mes previo al inicio de los síntomas.

$\mathrm{Al}$ ingreso se constatan los signos vitales temperatura axilar de $36,6^{\circ} \mathrm{C}$, frecuencia cardíaca de 116 latidos por minuto, tensión arterial de $80 / 50 \mathrm{mmHg}$, frecuencia respiratoria de 19 respiraciones por minuto y saturación de oxígeno del $99 \%$. Se registró una talla de 140 $\mathrm{cm}$ y peso de $59 \mathrm{~kg}$, con un índice de masa corporal de 30,1. Se observaron signos de deshidratación leve, mucosas semihúmedas, labios agrietados, lengua saburral e inyección conjuntival. El abdomen presentaba dolor difuso a la palpación profunda y se encontraron pequeñas máculas hipercrómicas en miembros inferiores. Se solicitaron estudios de laboratorio: hematocrito $36 \%$, hemoglobina $12,4 \mathrm{mg} / \mathrm{dl}$, recuento plaquetario $136000 / \mathrm{mm}^{3}$, recuento leucocitario $23100 / \mathrm{mm}^{3}$ (90\% neutrófilos, $3 \%$ eosinófilos, $6 \%$ leucocitos y $1 \%$ monocitos), ERS $75 \mathrm{~mm}$ en la primera hora, PCR $395 \mathrm{mg} / \mathrm{L}$, glucemia $81 \mathrm{mg} / \mathrm{dL}$, urea $118 \mathrm{mg} / \mathrm{dL}$, creatinina $1,39 \mathrm{mg} / \mathrm{dL}$, troponina T $79 \mathrm{ng} / \mathrm{L}, \mathrm{Na} 128 \mathrm{mEq} / \mathrm{L}$, $\mathrm{K} 3,2 \mathrm{mEq} / \mathrm{L}, \mathrm{Cl} 90 \mathrm{mEq} / \mathrm{L}$, estado ácido-base: $\mathrm{pH}$ 7,42, bicarbonato 19,5 $\mathrm{mmmol} / \mathrm{L}$, EB -4,2, ferritina $>2000 \mathrm{mg} / \mathrm{L}$, amilasa $174 \mathrm{U} / \mathrm{L}$, lipasa $1575 \mathrm{U} / \mathrm{L}$, fibrinógeno $682 \mathrm{ng} / \mathrm{dL}$, dímero D $3650 \mathrm{ng} / \mathrm{mL}$.

El electrocardiograma mostró elevación del ST en DIII y AVF. El ecocardiograma evidenció un leve aumento de refringencia del pericardio en la cara posterior diafragmática, compatible con pericarditis leve. La radiografía de tórax y la ecografía abdominal no mostraron alteraciones. $\mathrm{Al}$ ingreso, se realizó una expansión de volumen con solución fisiológica $(500 \mathrm{~mL})$, se tomaron muestras para dos hemocultivos y se indicó tratamiento antibiótico empírico con ceftriaxona en dosis de $1 \mathrm{~g}$ cada 12 horas por vía intravenosa (IV). Continuó con balance hídrico estricto por presentar insuficiencia renal aguda de origen

FIGURA 1. Definición de caso confirmado de síndrome inflamatorio multisistémico posCOVID-19 en pediatría del Ministerio de Salud de la Nación Argentina

\section{Definición de caso adaptada de la Organización Mundial de la Salud}

Niños y adolescentes de 0 a 18 años con fiebre mayor a 3 días:

Y dos de los siguientes:

A. Erupción cutánea o conjuntivitis bilateral no purulenta o signos de inflamación mucocutánea (oral, manos o pies).

B. Hipotensión o shock.

C. Características de disfunción miocárdica, pericarditis, valvulitis o anomalías coronarias (incluidos los hallazgos ecográficos o elevación de troponina/NT-proBNP).

D. Evidencia de coagulopatía (elevación de PT, PTT, dímero D).

E. Síntomas gastrointestinales agudos (diarrea, vómitos o dolor abdominal).

\section{Y}

Marcadores elevados de inflamación, como eritrosedimentación, proteína C reactiva o procalcitonina.

\section{Y}

Ninguna otra causa evidente de inflamación (incluidos la sepsis bacteriana y síndromes de shock estafilocócicos o estreptocócicos).

Fuente: Ministerio de Salud Argentina. Definición de caso de COVID-19. Buenos Aires: Ministerio de Salud de la Nación, 2020. Disponible en: https: / / www.argentina.gob.ar/salud/ coronavirus-COVID-19/definicion-de-caso 
prerrenal y pancreatitis, con disminución de los valores de urea y creatinina luego del aporte de volumen. Se solicitó un hisopado nasofaríngeo para PCR para SARS-CoV-2, con resultado negativo, y anticuerpos totales (IgM e IgG) para COVID-19 positivos (98 UI/L y $65 \mathrm{UI} / \mathrm{L}$ ). Con base en los criterios del Ministerio de Salud de la Nación adaptados de la Organización Mundial de la Salud ${ }^{10}$ (edad, fiebre de más de 3 días, erupción cutánea, conjuntivitis no purulenta, signos de inflamación mucocutánea, diarrea y dolor abdominal, pericarditis, elevación de reactantes de fase aguda, dímero $\mathrm{D}$, anticuerpos anti-SARSCoV-2 positivos) se interpreta el cuadro como un SIM-C (Figura 1).

A las 36 horas de internación continuó en regular estado general, febril, con 3 registros diarios de $38{ }^{\circ} \mathrm{C}$ a $39{ }^{\circ} \mathrm{C}$ y deposiciones diarreicas. Se decidió comenzar tratamiento con inmunoglobulina intravenosa (IGIV), única dosis de 1,3 g/ kg/día, y ácido acetilsalicílico (AAS) en dosis de $50 \mathrm{mg} / \mathrm{kg} /$ día por cumplir criterios de enfermedad de Kawasaki incompleta. A las 48 horas, se suspendió antibioticoterapia por hemocultivos negativos. Presentó buena respuesta clínica al tratamiento, con desaparición de la fiebre, mejora de cuadro grastrointestinal y disminución de los marcadores inflamatorios). A las 72 horas se disminuyó la dosis de AAS a dosis antiagregante, de $100 \mathrm{mg}$ / día.
Al cuarto día de internación presentó alteración del coagulograma (consumo de protrombina $-\mathrm{CP}-44 \%$, razón internacional normatizada-RIN-1,84, tiempo de protrombina $20 \mathrm{seg}$ ). Se indica la administración de vitamina K por vía intramuscular en dosis de $10 \mathrm{mg} /$ día por 3 días, con posterior normalización de los valores. Si bien presentó una mejora de los síntomas (sin fiebre y con disminución del dolor abdominal) y de los valores de reactantes de fase aguda e inflamatorios, las enzimas pancreáticas continuaron en ascenso, sin asociar nuevas manifestaciones (Figura 2). Se realizó una tomografía de abdomen, en la que se observó un aumento difuso del tamaño del páncreas, sin lesiones focales ni cambios en la grasa peripancreática, ni evidencia de compromiso trombótico. La colangiopancreatografía por resonancia magnética no mostró compromiso anatómico.

Las serologías para virus de EpsteinBarr, citomegalovirus, hepatitis A, B y C, inmunodeficiencia humana, parvovirus B $19 \mathrm{y}$ Mycoplasma pneumoniae fueron negativas.

Luego de descartar otras posibles causas de pancreatitis aguda, se asumió como una complicación inflamatoria del SIM-C. Al noveno día de internación, se indicó meprednisona en dosis de $1 \mathrm{mg} / \mathrm{kg}$ / día (60 mg totales) por vía oral, observando mejoría de los valores de enzimas

FIgURA 2. Evolución de los valores de lipasa y amilasa

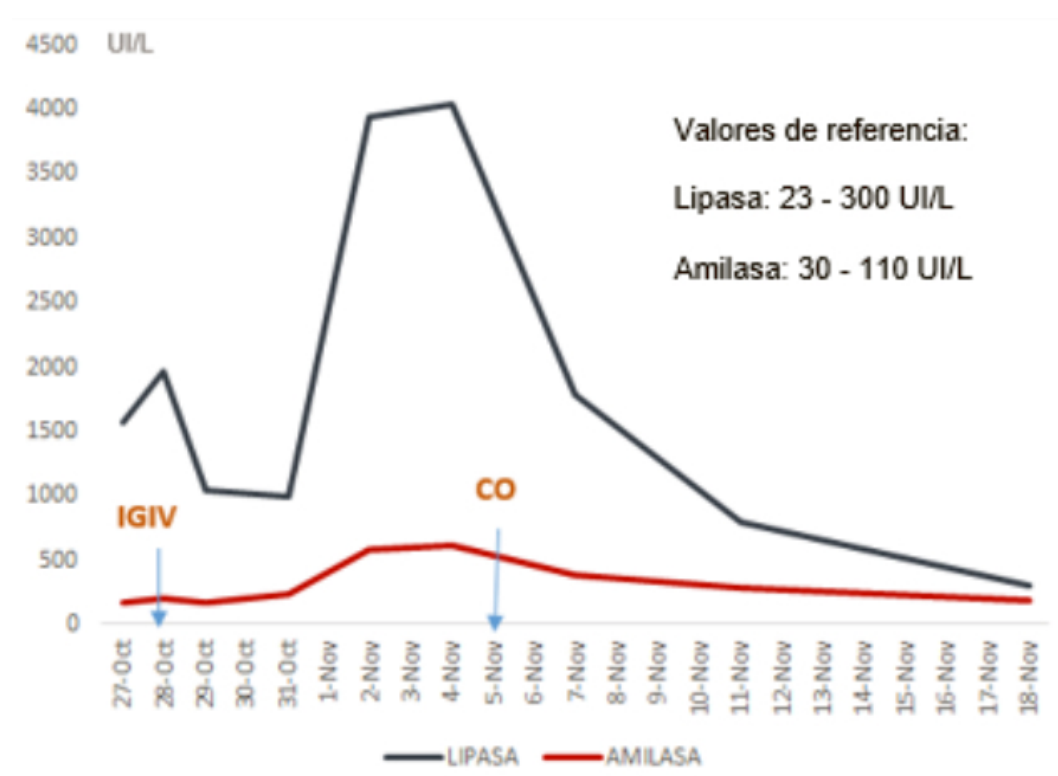

IGIV: tratamiento con inmunoglobulina humana intravenosa; CO: tratamiento con glucocorticoides vía oral. 
pancreáticas y parámetros de inflamación (Tabla 1). Se mantuvo la dosis de $60 \mathrm{mg} /$ día de meprednisona por 10 días, con descenso gradual (20\% cada 72 horas).

Se otorgó el alta hospitalaria luego de 11 días de internación con seguimiento ambulatorio por los servicios de pediatría, cardiología, gastroenterología y endocrinología, observando en los controles posteriores normalización de los valores de las enzimas pancreáticas y marcadores de inflamación (Cuadro 1). Se suspendió tratamiento antiagregante con control cardiológico y ecocardiograma normal a las 8 semanas.

\section{DISCUSIÓN}

Se describe el caso de una paciente con una forma inusual de presentación dentro del síndrome inflamatorio multisistémico postCOVID en pediatría. Las notificaciones de pancreatitis aguda en niños con SIM-C son escasas, a diferencia de lo que ocurre en adultos donde, en el contexto de infección aguda por SARS-CoV-2, se han informado más casos de pancreatitis. ${ }^{4}$ Según un estudio de cohorte retrospectivo que analizó a 11883 pacientes hospitalizados con COVID-19 de 12 hospitales de Estados Unidos, hubo 32 casos de pancreatitis aguda, el $69 \%$ de ellos idiopáticos, lo que mostró una prevalencia puntual del $0,27 \% .{ }^{5}$ Si bien aún no está del todo dilucidado la forma en que se produce el daño, la lesión pancreática podría ser causada por diferentes mecanismos: en forma directa por la invasión viral, por anomalías enzimáticas en el contexto de una enfermedad grave sin una lesión pancreática sustancial, por lesiones microvasculares y trombosis, como consecuencia del COVID-19, asociadas a shock e hipoperfusión gastrointestinal y, en los SIM-C, por la respuesta inflamatoria sistémica, evidenciada por el aumento de biomarcadores como la proteína $C$ reactiva, procalcitonina e interleucina $66^{6-8}$ En la paciente presentada se consideró, como causa más probable de pancreatitis aguda, la respuesta inflamatoria postCOVID-19, evidenciada por niveles elevados de reactantes de fase aguda, el tiempo de exposición al virus, el resto de la etiología negativa y la evolución posterior favorable con el abordaje terapéutico. ${ }^{9}$

Aunque al recibir tratamiento con inmunoglobulinas se evidenció mejoría clínica y de los parámetros inflamatorios, las enzimas pancreáticas continuaron en aumento. Esto, sumado al dolor abdominal y al aumento del tamaño del páncreas en tomografía, se interpretó el cuadro como pancreatitis aguda. ${ }^{10}$

En cuanto al tratamiento, la IGIV está recomendada para todos los pacientes que cumplan los criterios de enfermedad de Kawasaki completa o incompleta. ${ }^{11}$ En una notificación de caso de pancreatitis asociada a SIM-C, se administraron dos dosis de inmunoglobulina, con una respuesta favorable. ${ }^{12}$

TABLA 1. Evolución del laboratorio del paciente y el tratamiento instaurado

\begin{tabular}{|c|c|c|c|c|c|c|c|c|c|}
\hline \multirow[t]{2}{*}{$\begin{array}{l}\text { Días desde el } \\
\text { ingreso hospitalario }\end{array}$} & 0 & 1 & 2 & 4 & 6 & 8 & 11 & 22 & 29 \\
\hline & \multicolumn{2}{|c|}{$\begin{array}{c}\text { Previo al tratamiento } \\
\text { con IGIV y AAS }\end{array}$} & \multicolumn{4}{|c|}{$\begin{array}{l}\text { Luego del tratamiento } \\
\text { con IGIV y AAS }\end{array}$} & \multicolumn{3}{|c|}{$\begin{array}{l}\text { Luego del tratamiento } \\
\text { con corticoides }\end{array}$} \\
\hline GB/linfocitos (uL) & $\begin{array}{c}23120 / \\
1387\end{array}$ & $\begin{array}{c}19170 / \\
1534\end{array}$ & 15840 & $\begin{array}{c}13730 / \\
4120\end{array}$ & $\begin{array}{c}14730 / \\
3680\end{array}$ & $\begin{array}{c}10020 / \\
4400\end{array}$ & $\begin{array}{c}10990 / \\
2530\end{array}$ & $\begin{array}{c}11909 / \\
4330\end{array}$ & $\begin{array}{c}11210 / \\
5380\end{array}$ \\
\hline PCR (mg/L) & 395,1 & 323,7 & - & 37,5 & 17,6 & 7,7 & $<5$ & $<5$ & 6,4 \\
\hline ERS (mm en la $1^{\text {a }}$ hora) & 75 & 60 & - & 60 & 45 & 45 & 45 & 5 & \\
\hline Ferritina (ng/mL) & $>2000$ & $>2000$ & - & 704 & 635 & - & - & 247 & \\
\hline Troponina T (ng/mL) & 79 & 17,5 & - & 5,5 & - & - & - & - & \\
\hline Dímero D (ng/ml) & 3650 & 2000 & - & - & 1940 & 1210 & - & - & 280 \\
\hline Amilasa (UI/L) & 174 & 202 & 164 & 238 & 577 & 610 & 384 & 189 & 102 \\
\hline Lipasa (UI/L) & 1575 & 1974 & 1051 & 997 & 3947 & 4041 & 1786 & 310 & 185 \\
\hline
\end{tabular}

IGIV: inmunoglobulina intravenosa, AAS: ácido acetilsalicílico, GB: glóbulos blancos, PCR: proteína C reactiva, ERS: eritrosedimentación. 
El uso de esteroides, más a menudo metilprednisolona, para el tratamiento del SIM-C se ha informado con frecuencia en la literatura, con un rango de 49-73\%.13 La justificación del uso de los esteroides son sus propiedades antiinflamatorias y su uso frecuente tanto en EK como en otros trastornos inflamatorios. ${ }^{14}$ Si bien el SIM-C suele presentarse con mayor frecuencia en niños mayores y adolescentes, a diferencia de la EK, más prevalente en lactantes y preescolares, un tercio de los pacientes con SIM-C cumplen los criterios de Kawasaki, lo que dificulta su diagnóstico certero. ${ }^{12,14}$ Dentro de las manifestaciones clínicas del SIM-C se destacan las gastrointestinales y la disfunción miocárdica y los niveles elevados de reactantes de fase aguda, como en el caso presentado.

El Colegio Estadounidense de Reumatología recomienda, con grado de recomendación fuerte a moderada, el uso de corticoides en casos refractarios a la inmunoglobulina, en casos graves (en forma de pulsos) y como primera línea en todos los casos. ${ }^{13}$ La paciente tuvo una respuesta parcial a la administración de inmunoglobulina, ya que se evidenció mejora clínica y de parámetros de laboratorio, pero el cuadro de pancreatitis continuó desmejorando. Tras descartar otras posibles causas de esta afectación, se asume la pancreatitis aguda como complicación del SIM-C y se decide comenzar tratamiento con corticoides a $1 \mathrm{mg} / \mathrm{kg}$ y evaluar su duración según la evolución. La respuesta fue muy favorable, ya que las enzimas pancreáticas comenzaron su descenso a las 48 horas de haber comenzado la terapia y tuvieron una tendencia decreciente hasta llegar a valores normales.

\section{REFERENCIAS}

1. Pediatric Intensive CareSociety. PICSStatement: Increased number of reported cases of novel presentation of multisystem inflammatory disease. Londres, 27 de abril 2020. [Acceso: 15 de abril de 2021]. Disponible en: https: / / picsociety.uk/news / pics-statement-regarding-novelpresentation-of-multi-system-inflammatory-disease/

2. Feldstein LR, Rose EB, Horwitz SM, Collins JP, et al. Multisystem inflammatory syndrome in U.S. children and adolescents. N Engl J Med. 2020; 383(4):334-46.
3. Godfred-CatoS, Bryant B, Leung J, Oster ME, et al. COVID19-Associated Multisystem Inflammatory Syndrome in Children - United States, March-July 2020. MMWR Morb Mortal Wkly Rep. 2020; 69(32):1074-80.

4. Alves AM, Yvamoto EY, Marzinotto M, Teixeira A, et al. SARS-CoV-2 leading to acute pancreatitis: an unusual presentation. Braz J Infect Dis. 2020; 24(6):561-4.

5. Gonzalo-Voltas A, Fernández-Pérez-Torres CU, BaenaDíez JM. Pancreatitis aguda en paciente con infección por COVID-19. Med Clin (Barc). 2020; 155(4):183-4.

6. Inamdar S, Benias PC, Liu Y, Sejpal DV, et al. Prevalence, risk factors, and outcomes of hospitalized patients with coronavirus disease 2019 presenting as acute pancreatitis. Gastroenterology. 2020; 159(6):2226-8.e2.

7. Kabeerdoss J, Pilania RK, Karkhele R, Kumar TS, et al. Severe COVID-19, multisystem inflammatory syndrome in children, and Kawasaki disease: immunological mechanisms, clinical manifestations and management. Rheumatol lnt. 2021; 41(1):19-32.

8. Juhász MF, Ocskay K, Kiss S, Hegyi P, et al. Insufficient etiological workup of COVID-19-associated acute pancreatitis: A systematic review. World J Gastroenterol. 2020; 26(40):6270-8.

9. Chen N, Zhou M, Dong X, Qu J, et al. Epidemiological and clinical characteristics of 99 cases of 2019 novel coronavirus pneumonia in Wuhan, China: a descriptive study. Lancet. 2020; 395(10223):507-13.

10. Argentina. Ministerio de Salud. Manejo inicial del síndrome inflamatorio multisistémico en niños y adolescentes relacionados temporalmente al COVID-19 (SIM-C): recomendaciones. 31 de agosto 2020. [Acceso: 15 de abril de 2021]. Disponible en: https:// bancos.salud.gob.ar/sites / default / files / 2020-09/ covid19-manejo-inicial-del-sindrome-inflamatoriomultisistemico-en-ninios-y-adolescentes-relacionadostemporalmente-al-cvod19-sim-c.pdf

11. García Burriel JI, Vilar Escrigas P. Pancreatitis en el niño. Protocolos diagnóstico-terapéuticos de Gastroenterología, Hepatología y Nutrición Pediátrica SEGHNP-AEP. 2010:13542. [Acceso: 15 de abril de 2021]. Disponible en: https:/ / www.aeped.es/sites / default/files / documentos / pancreatitis.pdf

12. Stevens J, Brownell JN, Freeman AJ, Bashaw H. COVID-19associated multisystem inflammatory syndrome in children presenting as acute pancreatitis. J Pediatr Gastroenterol Nutr. 2020; 71(5):669-71.

13. Henderson LA, Canna SW, Friedman KG, Gorelik M, et al. American College of Rheumatology Clinical Guidance for multisystem inflammatory syndrome in children associated with SARS-CoV-2 and hyperinflammation in pediatric COVID-19: Version 1. Arthritis Rheumatol. 2020; 72(11):1791-1805.

14. Elias DM, McCrindle BW, Larios G, Choueiter NF, et al. Management of multisystem inflammatory syndrome in children associated with COVID-19: a survey from the international Kawasaki disease registry. CJC Open. 2020; 2(6):632-40. 